\title{
La reforma educativa en México ¿responde a la carencia de formación de lectores?: estudio de caso en la escuela primaria vespertina real del río, ciclo escolar 2011-2012
}

\author{
Leonardo Cíntora, Centro Universitario de Tijuana, Campus Mexicali, \\ Baja California, Mexico
}

\begin{abstract}
Resumen: La lectura de comprensión representa la herramienta fundamental para el desarrollo de habilidades y competencias en la educación básica de los alumnos, que utilizarán durante el transcurso de su vida escolar. Se ha demostrado que México se encuentra en una crisis en la formación de lectores, en este artículo se investigó a un grupo de primer grado de primaria en Mexicali Baja California, que trabajó con la reforma de educación básica que se implementó en el 2009 en el país, que excluye cualquier método de lecto-escritura en sus programas, tratándose de probar que esto es incorrecto para la situación socioeconómica actual de la región en este caso se complementó el trabajo con la método global de análisis estructural. Se les aplicó un instrumento de medición establecido por la Secretaría de Educación Pública aunado a un análisis con el software cientifico IBM SPSS statistics versión 20 , obteniendo un .948 de confiabilidad en Alfa de Cronbach. Se obtuvo la media general, además se realizaron correlaciones y diferentes interpretaciones de los datos recabados, resultado que la mayoría alcanza los estándares nacionales, al haber trabajado con el método de lecto-escritura y la reforma educativa.
\end{abstract}

Palabras Clave: comprensión Lectora, Estrategias y Situación Socioeconómica

\begin{abstract}
Reading comprehension is a fundamental tool for the development of skills and competencies in basic education of students who use throughout their school life. It has been shown that Mexico is in a crisis in the education of readers, in this article we investigated a group of first grade in Mexicali Baja California, who worked with the basic education reform that was implemented in 2009 in the country, which excludes any method of reading and writing in their programs, case to prove that this is wrong for the current socio-economic situation of the region in this case the work was complemented with the overall method of structural analysis. They were given a measuring instrument established by the Ministry of Education together with an analysis in IBM SPSS Statistics scientific software version 20, obtaining 948 of Cronbach's alpha reliability. The mean overall, and correlations were performed and different interpretations of the data collected, the majority reaches a result that national standards, having worked with the method of literacy and education reform.
\end{abstract}

Keywords: Reading Comprehension, Strategies and Socioeconomic Status

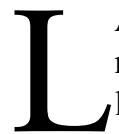

A SOCIEDAD MEXICANA ha demandado una educación de calidad a nivel mundial, fruto de esa exigencia se crea el Instituto Nacional para la Evaluación de la Educación (INEE) en 2002 (Backhoff, Sánchez, Peón y Andrade, 2010), pero ¿cómo está evaluado México ante los estándares internacionales? Actualmente México se encuentra por abajo del promedio alcanzado por los países miembros de la OCDE, dos de cada 5 estudiantes mexicanos de 15 años (39\%) se ubica en los niveles inferiores de

Revista Internacional de Educaci ón y Aprendizaje

Volumen 1, 2013, http://sobrelaeducacion.com/revistas/coleccion/, ISSN 2255-453X

(C) Global Knowledge Academics. Leonardo Cíntora

Todos los Derechos Reservados. Permisos: soporte@gkacademics.com

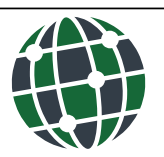


desempeño (1a y 1 b y por debajo de 1); estos jóvenes poseen competencias lectoras insuficientes para aprender y participar de forma eficaz y productiva en situaciones de la vida real. En tanto, $54 \%$ de los alumnos mexicanos se coloca en los niveles 2 y 3, lo que refleja que poseen competencias mínimas para comprender, emplear y reflexionar lo que leen y usarlo en situaciones reales. Sólo 6\% de los jóvenes mexicanos de 15 años se ubican en los niveles más altos de desempeño en lectura (4 a 6). La comparación entre los resultados de México en PISA 2000 y 2009 indica que disminuyó la proporción de estudiantes en los niveles bajos (1 y debajo de 1), pasando de 44\% a 39\%. Pero también disminuyó la proporción de estudiantes en los niveles más altos (4 y superior), de $7 \%$ a $6 \%$. Asimismo, se incrementó el porcentaje de estudiantes en los niveles medios ( 2 y 3), que pasó de 49\% a 54\%. (OCDE, PISA 2009)

Estas estadísticas sitúan a México en desventaja para competir con un sistema educativo que responda a las necesidades y estándares mundiales. Sin embargo, existen voces que reclaman el por qué se debe de calificar a México con países que cuentan con mayores presupuestos educativos o condiciones socio-económicas más elevadas. Por lo que ahora se presentan los resultados de la evaluación nacional llamada ENLACE (Evaluación Nacional de Logro Académico en Centros Escolares), la cual es una prueba del Sistema Educativo Nacional que se aplica a planteles públicos y privados del país.

Como el trabajo se centrará en un grupo específico se reducirá de manera paulatina el antecedente de información, por lo que dentro de los resultados de enlace únicamente se presenta el resultado de Baja California, el resultado global en español dentro de la categoría de escuela primaria general el $61.8 \%$ se encuentra entre nivel insuficiente y elemental, es decir sólo el $31.4 \%$ obtuvo un nivel bueno y únicamente el $6.8 \%$ un nivel excelente. Con esto se observa que la mayoría de los alumnos evaluados no desarrollan suficientes competencias en lectura de comprensión (página oficial de ENLACE http://www.enlace.sep.gob.mx/ba/) y cómo se encuentra evaluada la Escuela Primaria Real del Río vespertina en la prueba ENLACE de los últimos 3 años, a continuación los resultados:

\begin{tabular}{|c|c|c|c|c|c|c|c|c|c|c|c|c|c|}
\hline & & \multicolumn{3}{|c|}{ INSUFICIENTE } & \multicolumn{3}{|c|}{ ELEMENTAL. } & \multicolumn{3}{|l|}{ BUENO } & \multicolumn{3}{|c|}{ EXCELENTE } \\
\hline & & Escuela & Entidad & Pais & Escuela & Entidad & Pais & Escuela & Entidad & Pais & Escuela & Entidad & Pais \\
\hline \multirow[b]{3}{*}{$3^{2}$} & 2011 & $0.00 \%$ & $14.00 \%$ & $11.80 \%$ & $4.80 \%$ & $4150 \%$ & $38.40 \%$ & $66.70 \%$ & $33.70 \%$ & $36.00 \%$ & $28.60 \%$ & $10.80 \%$ & $13.80 \%$ \\
\hline & 2010 & $22.60 \%$ & $1430 \%$ & $11.60 \%$ & $43.50 \%$ & $41.80 \%$ & $41.60 \%$ & $30.60 \%$ & $34.70 \%$ & $36.70 \%$ & $3.20 \%$ & $9.20 \%$ & $10.10 \%$ \\
\hline & 2009 & $19.00 \%$ & $13.80 \%$ & $1300 \%$ & $54.80 \%$ & $41.40 \%$ & $40.40 \%$ & $23.80 \%$ & $39.10 \%$ & $39.70 \%$ & $2.40 \%$ & $5.70 \%$ & $6.80 \%$ \\
\hline \multirow[b]{3}{*}{$4^{*}$} & 2011 & $14.30 \%$ & $16.70 \%$ & $16.30 \%$ & $57.10 \%$ & $4250 \%$ & $40.80 \%$ & $25.40 \%$ & $32.00 \%$ & $3220 \%$ & $3.20 \%$ & $8.80 \%$ & $10.70 \%$ \\
\hline & 2010 & $30.40 \%$ & $19.00 \%$ & $19.20 \%$ & $52.20 \%$ & $46.10 \%$ & $44.70 \%$ & $17.40 \%$ & $28.20 \%$ & $28.70 \%$ & $0.00 \%$ & $6.70 \%$ & $7.40 \%$ \\
\hline & 2009 & $25.00 \%$ & $18.00 \%$ & $1690 \%$ & $60.70 \%$ & $51.70 \%$ & $50.90 \%$ & $1430 \%$ & $26.60 \%$ & $27.50 \%$ & $0.00 \%$ & $3.70 \%$ & $4.70 \%$ \\
\hline \multirow{5}{*}{$5^{*}$} & 2011 & $9.80 \%$ & $1250 \%$ & $12.60 \%$ & $63.40 \%$ & $57.50 \%$ & $5620 \%$ & $26.80 \%$ & $2790 \%$ & $28.40 \%$ & $0.00 \%$ & $2.10 \%$ & $2.80 \%$ \\
\hline & 2010 & $6.70 \%$ & $1530 \%$ & $16.10 \%$ & $76.70 \%$ & $5390 \%$ & $53.20 \%$ & $16.70 \%$ & $27.40 \%$ & $27.30 \%$ & $0.00 \%$ & $330 \%$ & $3.40 \%$ \\
\hline & 2009 & $15.60 \%$ & $14.80 \%$ & $15.50 \%$ & $68.90 \%$ & $54.80 \%$ & $53.60 \%$ & $15.00 \%$ & $27.30 \%$ & $27.70 \%$ & $0.00 \%$ & $3.10 \%$ & $3.20 \%$ \\
\hline & 2011 & $16.70 \%$ & $11.70 \%$ & $11.70 \%$ & $60.00 \%$ & $47.20 \%$ & $4450 \%$ & $23.30 \%$ & $35.70 \%$ & $36.60 \%$ & $0.00 \%$ & $540 \%$ & $7.20 \%$ \\
\hline & 2010 & $16.20 \%$ & $9.00 \%$ & $9.70 \%$ & $64.90 \%$ & $50.20 \%$ & $48.10 \%$ & $18.90 \%$ & $37.70 \%$ & $38.80 \%$ & $0.00 \%$ & $3.20 \%$ & $3.40 \%$ \\
\hline $6^{\circ}$ & 2009 & $0.00 \%$ & $19.00 \%$ & $1650 \%$ & 75.0006 & $5420 \%$ & $53.20 \%$ & $15.00 \%$ & $25.00 \%$ & $27.50 \%$ & $1000 \%$ & $1.80 \%$ & $2.90 \%$ \\
\hline
\end{tabular}

\section{ESPAÑOL}

Con estos resultados internacionales, nacionales y locales se prende un foco de alarma que indica la deficiencia en el trabajo con la lectura de comprensión. A qué se refiere esto en específico, a comprobar que si los maestros trabajaran con un sistema de enseñanza de lectoescritura analítica, en específico el método Global de Análisis Estructural. Los resultados en las evaluaciones mejorarían considerablemente. ¿Por qué creer en esto?, las generaciones que entran a los rangos de evaluación de los exámenes de PISA y ENLACE fueron alfabetizados con métodos de origen sintético en donde predomina la memorización y no el análisis, 
ya que los docentes no fueron actualizados con métodos analíticos, y es un círculo vicioso que se está repitiendo en la actualidad, con una reforma a la educación básica en el año 2009 en la cual las actualizaciones consisten en informar de los cambios de estructura de programas y contenidos pero no de metodología de los primeros grados para beneficiar la comprensión lectora.

La lectura implica la interacción entre la información visual que se percibe a través del sistema visual y la información que ya se tiene disponible de él. En un acto de lectura puede haber intercambio y una relación directamente proporcional entre los dos tipos de información. Por ello es importante tomar en cuenta que durante la educación básica se busca favorecer el éxito o logro de la lectura comprensiva, ya que en la medida en que el docente reconozca que aunque el procedimiento puede ser similar, existen múltiples determinantes que inciden en la forma de aprender a leer. La lectura es un proceso que no se reduce a conocer símbolos, palabras, oraciones y partes abstractas del lenguaje que son objetos de estudio, leer consiste esencialmente en procesar el lenguaje y construir significados. Se sabe de la existencia de diversos métodos para el aprendizaje de la lectura, los cuales se engloban en dos grandes grupos: sintéticos y analíticos. Los sintéticos parten del elemento menor para integrar el todo, o bien, se caracteriza porque proceden por composición, es decir, pasando de las partes al todo. Este método insiste en la correspondencia entre lo oral y lo escrito, entre el sonido y la grafía, "desligándose de la enseñanza del lenguaje oral." (López y Álvarez, 1991, p.131). Y están los analíticos o globales, que se definen como los que inician de lo general a lo particular, surgida como una reacción del aprendizaje sintético, apoyada en los descubrimientos psicológicos de Ovide Decroly, y sobre todo en el sincretismo y la percepción global del niño.

Con la reforma a los planes de estudio de Educación básica de 1993 se pretendía introducir de lleno el constructivismo como base teórica del trabajo del docente, pero en las escuelas normales no se les daba bases sólidas a los futuros docentes con las cuales enfrentar el problema de la enseñanza de la lectura, por lo que se empezó a dar un mezcla de métodos que en muchas ocasiones se contradecían didácticamente hablando y creaban una confusión en los alumnos en la adquisición de la lectura tal y como se demuestra en un estudio realizado por la Dra. Rosa María Acosta Luévano (2007). Lo que el constructivismo plantea es la formación del conocimiento situándose en el interior del sujeto, es decir, "el sujeto construye el conocimiento de la realidad, ya que no puede ser conocida en sí misma, sino a través de los mecanismos cognitivos de que se dispone" (Araya, Alfaro y Andonegui, 2007, p.77). Con esto se suponía que los docentes debían trabajar con situaciones que pudieran experimentar en la vida real sus alumnos o que estuvieran lo más a pegadas a la misma, para que así los alumnos pudieran relacionar lo visto en la escuela con su realidad y transformarla para crear esos nuevos conocimientos.

Pero que sucedía en realidad, que ante la falta de actualización los docentes continuaban con su enseñanza bajo métodos como el silábico o el fonético, ambos de marcha sintética pues eran con los que ellos fueron alfabetizados. A la par con esa reforma surge la propuesta 03 o Metodología integradora, la cual tenía como misión fortalecer el aprendizaje de la lectura y la escritura en base a la concepción de la lectura como sistema comprensivo, no en el descifrado, en la que el niño expresa por escrito sus ideas. La propuesta estaba fundamentada en la teoría de Jean Piaget que consideraba al niño como un sujeto que busca adquirir conocimientos activamente, interactuando con el mundo que lo rodea, y la autora de esta propuesta era Emilia Ferreiro (1988). Pero nunca se le dio seguimiento a pesar de que los 
libros de texto estaban diseñados bajo este sistema. En el plan y programas de estudio de 1993 la premisa era construir en los alumnos aprendizajes significativos, pero los docentes desconocían la teoría que fundamentaba ese concepto y los autores que respaldaban dicho conocimiento. Esta definición tiene sus inicios más reconocidos con el psicólogo David Ausubel, rechaza el supuesto piagetiano de que solo se entiende lo que se descubre, ya que también puede entenderse lo que se recibe. "Un aprendizaje es significativo cuando puede relacionarse, con lo que el alumno ya sabe" (Ausubel, 1976). En México el sistema de educación básica está centralizado, es decir, los proyectos, adecuaciones curriculares, reformas y contenidos se deciden sin tomar en cuenta necesidades y situaciones que surgen por distintas circunstancias en las diferentes regiones o estados de la república. Debería haber flexibilidad y participación de los diferentes actores políticos de cada entidad para tomar acciones que respondan a las demandas que presenta el alumnado de hoy en día. En lo que respecta al tema de las reformas en los sistemas educativos, en México se ha estado trabajando por sexenios, dejando roto el enlace que debería darle continuidad a los programas educativos, lo que ha creado un vacío de fundamentos pedagógicos y filosóficos que den certidumbre a los docentes de cómo aplicar dichas reformas en su trabajo diario dentro del aula. Por lo anterior muchos docentes se han tenido que aferrar a la escuela tradicional y sus métodos, pues es de la forma que les enseñaron a ellos en su época estudiantil, y es de la única que tienen fundamentos.

Ante las evidencias de atraso en el sistema educativo mexicano y las evaluaciones internacionales y nacionales que se llevaron a cabo en la primera década del siglo XXI aparece la reforma de los planes y programas de Educación Básica en el año 2009, con una serie de cambios curriculares, la reorganización de contenidos, y la creación de un perfil del estudiante egresado de educación básica. Pero en lo que se refiere a la enseñanza de la lectura también aparecen cambios. Por ejemplo, desaparece el libro de lecturas para alumnos de primero durante los años de 2009 y 2010, además de que las actualizaciones a los docentes referentes a esta reforma eran para que conocieran el material, la organización de los contenidos y los nuevos conceptos que se manejarían en la literatura de la misma. Pero en ningún momento apareció la actualización para trabajar la lecto-escritura con el primer grado de primaria. En la guía para el maestro de los programas de estudio 2011, se encuentra la siguiente información, "al concluir el tercer grado de primaria, entre 8 y 9 años de edad, se sientan las bases para garantizar el éxito educativo porque al aprender a leer y escribir en un contexto de alfabetización inicial los alumnos tienen la posibilidad de emplear el lenguaje como herramienta de comunicación para seguir aprendiendo"(SEP, 2012, p.17), al momento de explicar la didáctica en que se basa maneja conceptos y estudios que carecen de información sobre dónde los hicieron o bajo qué circunstancias, pero no existe hasta la fecha una verdadera capacitación para saber cómo trabajar esos conceptos de los que se hace mención. Por lo que no se entiende el por qué hacer reformas sin haber primero hecho de manera integral el trabajo con los planes y programas anteriores, y ahora iniciar los docentes una labro dentro de las aulas sin fundamento teóricos ni actualización en sus dinámicas y estrategias de trabajo que respondan a las necesidades que establece la Reforma Integral de Educación Básica 2009.

En los nuevos programas 2011 aparecen una serie de conceptos que van acordes con las investigaciones mundiales actuales, tales como el trabajo por proyectos y bajo situaciones comunicativas en donde los alumnos y su docente interactúen entre ellos dentro de ambientes de aprendizaje que faciliten dichas actividades, la capacidad lectora consiste en la compren- 
sión, el empleo y la reflexión personal a partir de textos escritos con el fin de alcanzar las metas propias, desarrollar el conocimiento el potencial personal y de participar en la sociedad (OCDE-INCE, 2000) La escuela te enseña a leer, reconocer un sistema de representaciones escritas, pero no significa que se aprenda a leer (Carrasco, 2003). El problema principal de la reforma radica en la desarticulación entre la base docente y los expertos que elaboran los contenidos, cuando los programas son aplicados por profesionales ajenos al aula o incluso al centro educativo no logran crear ambientes de aprendizaje. Cuando el profesor es el que lo implementa la limitación estriba en la falta de conocimiento teórico y procedimental (De la Mata, 2003). En los docentes se ha creado un barrera de resistencia a las actualizaciones por parte de los sistemas de formación continua, debido a la falta de apego a la realidad que se vive en las escuelas, provocando que haya una escasa presencia en las aulas de actividades orientadas a instruir en la comprensión de textos (Madariaga, Chireac y Goñi, 2009).

Dentro de esa desarticulación, resulta alarmante que la reforma de planes y programas no iniciara o se empatara con el sistema de normales, encargadas de la formación docente. Los egresados como Licenciados en Educación Primaria en la actualidad desconocen cualquier metodología para la enseñanza de la lecto-escritura, creando un círculo vicioso en donde los estudiantes durante sus prácticas de campo copian o memorizan los métodos de maestros en servicio, quienes a su vez fueron formados con programas de más de 20 o 25 años de antigüedad. Lo anterior se reproduce en otros países por ejemplo cuando se trataba de explicar el bajo nivel alcanzado en el estudio de la formación de educadores de Párvulos en Chile (García-Huidobro, 2006), quien concluyó que los programas existentes incluían contenidos limitados y poco actualizados acerca del aprendizaje oral y escrito. Esta conclusión es consistente con las limitaciones encontradas en el programa Head Start desarrollado en los Estados Unidos de América; en relación con el conocimiento de los profesores respecto al aprendizaje de la alfabetización temprana (Powell, Diamond, Bojczyk y Gerde, 2008) lo que provoca que los estudiantes y futuros docentes se limiten a una o varias estrategias, siendo escasos los que combinan las de comprensión y metacomprensión (Martínez y Madariaga, 2006)

Dentro del Plan y Programas de Estudio 2011 aparecen recursos que favorecerán a desarrollar la lectura de comprensión. Primero los docentes deben planear sus actividades, pues es un proceso fundamental en su ejercicio ya que contribuye a plantear acciones para orientar la intervención del maestro hacia el desarrollo de competencias. El caso de la lectura de comprensión se debe de trabajar a través de un proceso transversal (Arroyo, Paz, Gazca y Orozco, 2010), articulando actividades dentro de todas las asignaturas que favorezcan a dicho fin. Una vez elaborada la planeación, deberá propiciar el docente aplicarla dentro de ambientes de aprendizaje propicios, los cuales son escenarios construidos para favorecer de manera intencionada las situaciones de aprendizaje. Constituye la edificación de situaciones de aprendizaje en el aula, en la escuela y en el entorno, pues el hecho educativo no sólo tiene lugar en el salón de clases, sino fuera de él para promover la oportunidad de formación en otros escenarios presenciales y virtuales. Para diseñar un ambiente de aprendizaje, el profesor debe tomar en cuenta que las tecnologías de la información y la comunicación están cambiando radicalmente el entorno en el que los alumnos aprendían.

El Plan y Programas de Estudio 2011 para el primer grado de primaria propone que las situaciones de aprendizaje sean el medio por el cual se organice el trabajo docente, a partir de planear y diseñar experiencias que incorporen el contexto cercano de los niños y tienen como propósito problematizar eventos del entorno próximo, algo parecido a lo que establecía 
Vygotsky con su andamiaje y la Zona de Desarrollo Múltiple (1978). Esas situaciones se pueden desarrollar dentro de dos modalidades, puede ser por proyectos, los cuales se definen como un conjunto de actividades sistemáticas e interrelacionadas para reconocer y analizar una situación o problema y proponer posibles soluciones. Brindan oportunidades para que los alumnos actúen como exploradores del mundo, estimulen su análisis crítico, propongan acciones de cambio y su eventual puesta en práctica; los conduce no sólo a saber indagar, sino también a saber actuar de manera informada y participativa (SEP,2012). La otra modalidad son las secuencias didácticas, y se definen como actividades de aprendizaje organizadas que responden a la intención de abordar el estudio de un asunto determinado, con un nivel de complejidad progresivo en tres fases: inicio, desarrollo y cierre. Presentan una situación problematizadora de manera ordenada, estructurada y articulada. Para la lectura de comprensión es importante el trabajo colaborativo, por ejemplo que entre los compañeros sean socios de lectura (Flint, 2010), para que se funcional este trabajo debe ser inclusivo, entendiendo esto desde la diversidad, lo que implica orientar las acciones para que en la convivencia, los estudiantes expresen sus descubrimientos, soluciones, reflexiones, dudas, coincidencias y diferencias a fin de construir en colectivo. Se ha mencionado que la lectura de comprensión es la base académica para que cualquier alumno transite con seguridad y respaldo el resto de su vida escolar. Para ello se necesita que todos los agentes educativos colaboren (López, 2009). En México, a pesar de que el periodo 2001- 2006 se ha concebido como aquél para promover la lectura como política gubernamental, y en particular como política del sector educativo con el programa: México, un país de lectores (Díaz Barriga, 2001) aún estamos lejos de lograrlo.

Se tendrá que poner atención a tres conceptos que vienen marcando el camino de las recientes investigaciones, como la alfabetización temprana, alfabetización emergente y la meta-comprensión. Para poder a partir de eso diseñar un proceso estratégico acorde con las necesidades reales de un sistema educativo en determinado país. La principal preocupación de un docente en educación básica es el desarrollar en sus alumnos el mayor número de competencias y habilidades que le den certeza para transitar durante su vida académica. Por consiguiente, una de las prioridades de un gobierno debe ser la educación de sus ciudadanos, pero no de manera central como hasta hoy se viene realizando en México, un ejemplo sería como en Australia donde los planes de estudio son estatales (Hipwell y Klenowski, 2011). El dominio de la lengua es complejo y continúa desarrollándose durante toda la vida, se ha comprobado que sus fundamentos se adquieren durante los años previos al inicio de la enseñanza formal de la lectura y la escritura (Scarborough, 2001). La evidencia científica reciente destaca la importancia de la alfabetización temprana como fundamento del aprendizaje lector posterior, especialmente en niños de riesgo social (Villalón, Forster, Cox, Rojas, Valencia y Volante, 2011). Se hará énfasis en ese sector de la sociedad pues la escuela donde se desarrolló la investigación está dentro de los parámetros de riesgo social, por su nivel socio-económico y cultural.

El desarrollar habilidades de lenguaje temprano se ve como la puerta de entrada para generar mejoramientos significativos y perdurables (Abadzi, 2007), por lo anterior en los planes y programas de estudio de primer grado en México se le dedica un apartado especial a la alfabetización inicial, pero al analizarse se encuentra la primera contradicción con varios estudios internacionales, que consiste en señalar que para aprender a leer y escribir no existe una edad ideal, siendo que la alfabetización temprana ha mostrado resultados que fungen como mecanismos para romper el círculo de pobreza en el caso de la población con riesgo 
social (Justice, Mashburn, Hamre y Pianta, 2008) por tanto, al ser México un país con alto nivel de pobreza no se debe dejar tan amplio el margen temporal para iniciar con la alfabetización temprana. Los niños que crecen en un entorno alfabetizado no esperan hasta el inicio de la enseñanza escolar formal para explorar las características de la lectura y la escritura (Tolchinsky, 2006). Pero, cuál es la realidad de los alumnos que se reciben en las escuelas primarias públicas en situación de riesgo como es el caso de la institución investigada. Los alumnos no tienen apoyo familiar o sus entornos no son alfabetizadores, pues carecen de detonadores que los motiven dentro de sus hogares o peor aún, sus padres o hermanos tienen preparación trunca en algún punto medio de su educación básica, y la herencia también llega a ser determinante en el éxito escolar. (Taylor y Schatschneider, 2010) Llegan al primer año con un vocabulario pobre, el cual es determinante para el desarrollo de comprensión lectora (Shaw y McMillion, 2011). Con estas evidencias se vuelve a comprobar que en este tipo de escuelas no podemos aplicar la metodología igual que instituciones que reciben alumnos que tuvieron estimulación desde su hogar y con un vocabulario más amplio.

Es imperativo que en escuelas donde se reciben niños con los rezagos antes mencionados se enfatice el trabajo analítico de nuestro alfabeto, es decir, un meta-conocimiento o pensar acerca de lo que se sabe (Flavell, 1996), se ha establecido una asociación entre determinadas prácticas pedagógicas y el nivel de aprendizaje alcanzado por los niños, por ejemplo la enseñanza del código y el reconocimiento de letras y palabras (Connor, Morrison y Slominski, 2006), el conocimiento fonológico es clave en el desarrollo de la lectura en niños de primer grado (Araya, 2009). El conocimiento fonológico se define como la habilidad del alumno para tomar conciencia y manipular los elementos más simples del lenguaje oral como son las sílabas y los fonemas, facilitando el proceso inicial de lectura (Ramos, 2004). El programa de primer grado no señala que los niños deban leer de manera convencional al concluir el primer año de primaria, sino que busca ponerlos en contacto con los textos y que comiencen a leer y escribir, en este caso, con apoyo del docente, ya que se considera el segundo período de la educación básica (primero, segundo y tercero) para el proceso de adquisición de la lectura y la escritura de forma convencional. El papel del docente es fundamental para llevar a cabo una alfabetización temprana, en el caso de los planes y programas de estudio 2011 en México, establece que a la actual propuesta curricular no se le adscribe un método específico para enseñar a leer y escribir. Lo anterior no significa que se carezca de una propuesta de intervención docente, pero se menciona que las propuestas están basadas en los estudios psicolingüísticos donde las estrategias de intervención están adaptadas a las necesidades de los alumnos, y es el docente el que elige la forma de trabajar, pero el docente no tiene esas opciones para trabajar. En el estudio predictivo en el que se evaluó un conjunto amplio de variables pre-lectoras al inicio de la educación infantil, el mayor porcentaje de la varianza en la lectura de primero básico fue aportado por la identificación de las letras del alfabeto (Vellutino y Scanlon, 2002). La revisión de estudios de valor predictivo del conocimiento del alfabeto al inicio de la educación infantil lo sitúa como el predictor más potente, y en algunos casos, como el único del aprendizaje formal de la lectura (Foulin, 2005). Ante tales evidencias por qué negarle a los docentes en su formación una enseñanza de metodologías analíticas de lecto-escrituras para el caso en que atiendan a alumnos en situación de riesgo social. Si bien es cierto que las tendencias mundiales marcan el camino de la individualidad de los alumnos y sus procesos de construcción de su propio conocimiento, se elaboran programas estándares para escenarios ideales, pero qué hacer en contextos sociales donde la desintegración familiar, las necesidades económicas y la falta de escolaridad son las pautas 
que rigen la vida diaria de los alumnos. La lectura emergente es antes de ingresar a primaria (Smith, Jiménez y Ballesteros, 2004). En países que muestran más avance en evaluaciones internacionales tales como los Estados Unidos de América, se han diseñado programas de intervención en el contexto familiar y en los centros educativos, y al diseño de políticas públicas orientadas a promover el desarrollo de los niños menores de 6 años, especialmente en la población de mayor riesgo (Zill y Resnick, 2006). Pero en el sistema educativo mexicano, todos los programas son diseñados para características generales y sin tomar en cuenta particularidades que tiene cada población.

Se han identificado intervenciones efectivas para promover la alfabetización emergente, que incluye tanto la adquisición de destrezas de decodificación como el desarrollo del lenguaje oral (National Early Literacy Panel, 2008) es decir, los planes y programas para primer grado se encuentran flotando entre la modernidad pero dejando de lado cuestiones básicas que podrían formar esa amalgama entre lograr la alfabetización con un desarrollo de comprensión lectora. En un estudio longitudinal realizado por Bravo, Villalón y Orellana (2006) en niños con riesgo social de $1^{\circ}$ a $4^{\circ}$ básico, se encontró que el conocimiento del alfabeto fue el predictor de mayor peso al término de los cuatro cursos del primer ciclo de educación básica estudiados. Dentro de las recomendaciones que se dan a los docentes en los planes y programas del primer grado en la lectura en voz alta, que al igual como la lectura compartida han demostrado ser estrategias efectivas para desarrollar competencias lectoras, aunque su efectividad depende de que los educadores ayuden a los niños a avanzar desde sus conocimientos previos (MacKeown y Beck, 2006), pero ¿qué hacer cuando esos conocimientos previos están limitados?, la respuesta es simple, actuar. En un estudio de prácticas de enseñanza de la lectura en escuelas que atendían niños con riesgo social, los mejores resultados fueron alcanzado en las escuelas que dedicaban mayor tiempo a las actividades formales de aprendizaje, se leían cuentos y se trabajaba en un nivel más avanzado de conocimiento de letras y palabras (Eyzaguirre y Fontaine, 2008). De igual forma en un estudio realizado en Costa Rica se dividió a un grupo de primer año en tres subgrupos dependiendo de su nivel de conocimiento de las letras del alfabeto, la fonología y la escritura emergente, el grupo superior tuvo un desempeño lector significativamente más alto que los otros subgrupos (Bravo y colaboradores, 2006). Lo anterior fortalece la premisa que defiende que a los alumnos de primer grado de la escuela Primaria Real del Río vespertina en Mexicali, Baja California; se les debe de dar un sustento teórico en el conocimiento de sus sistema de escritura y lectura para que a partir de eso, ellos partan en la construcción de sus propios conocimientos y competencias. Actualmente en Mexicali se está impulsando el pase automático de primero, segundo y tercer grado de primaria en educación básica, con el argumento de que esos ciclos conforman un solo proceso, lo cual no está del todo errado, ¿pero acaso todos los alumnos se desarrollan igual?, o ¿adquieren las competencias suficientes para enfrentar la segunda fase de su proceso de adquisición de lectura de comprensión? En Costa Rica en el año 2006 un total de 13, 679 alumnos, es decir, el 8.3\% de los estudiantes de primer grado de primaria tuvieron que repetir el año escolar (Informe de la Nación, 2007). Lo anterior no significa que se deba de reprobar a todo aquel alumno de primer año que no desarrolle la lectoescritura, pero si tener en cuenta que se debe cumplir con cierto grado de alfabetización temprana, conocimiento del alfabeto y conciencia fonética. Qué establece el Plan y Programas de Estudio 2011 en el área de evaluación. Se debe aplicar a lo largo de todo el desarrollo del proyecto didáctico: al inicio, durante el proceso y el final del mismo. Por esta razón se proponen tres tipos de evaluación (diagnóstica, formativa y sumativa). La 
primera se utiliza para determinar el punto de partida, así como para tener un parámetro que permita valorar los avances en el proceso educativo. La evaluación formativa es el conjunto de acciones mediante las cuales se obtienen datos que permiten valorar el avance de los alumnos con respecto a los aprendizajes esperados o identificar los obstáculos que enfrentan al realizar las distintas actividades propuestas. Finalmente la evaluación sumativa o final permite saber si se lograron los propósitos planteados al inicio del proyecto. En conclusión el docente de primer grado tiene que realizar todo un proceso sistemático y continuo a lo largo del ciclo escolar para poder decidir de una manera justificada si su alumno aprueba o reprueba el año escolar.

La presente investigación es de metodología cuasiexperimental pues no tiene grupo de control y tampoco se hizo con aleatoriedad. Además el tipo de trabajo es de investigación acción pues tanto el investigador como el grupo son considerados parte del Universo de trabajo, estando a un mismo nivel de participación, además de ser una labor de intervención, pues como docente se fue aplicando una metodología a la par de la establecida por la Secretaría de Educación Pública. Los resultados de la encuesta se analizarán bajo un trabajo cuantitativo.

Hipótesis. La aplicación del método global de análisis estructural integrado con la didáctica de la Reforma Integral de Educación Básica está asociado con el logro de los estándares nacionales de habilidad lectora.

Para poder probar la hipótesis antes mencionada se trabajó con cuatro mediciones durante el ciclo escolar, utilizando los propios estándares, parámetros y definiciones que establece la Secretaría de Educación Pública. A continuación se presentan los estándares de habilidad lectora establecidos:

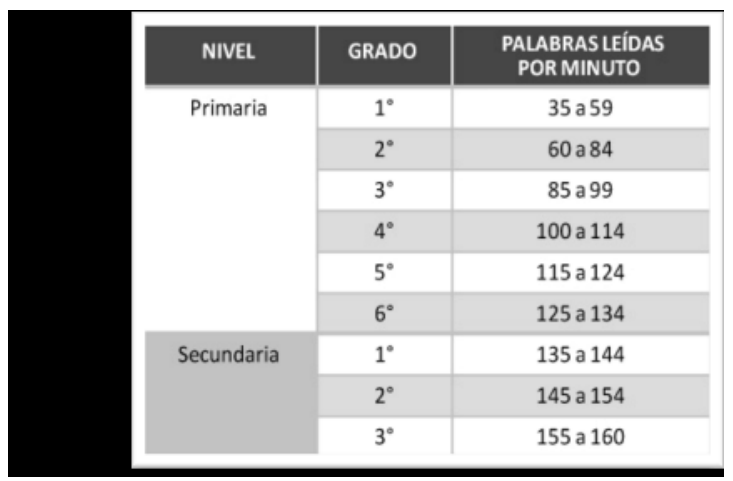

Fuente: http://www.sep.gob.mx/work/appsite/lectura.pdf 


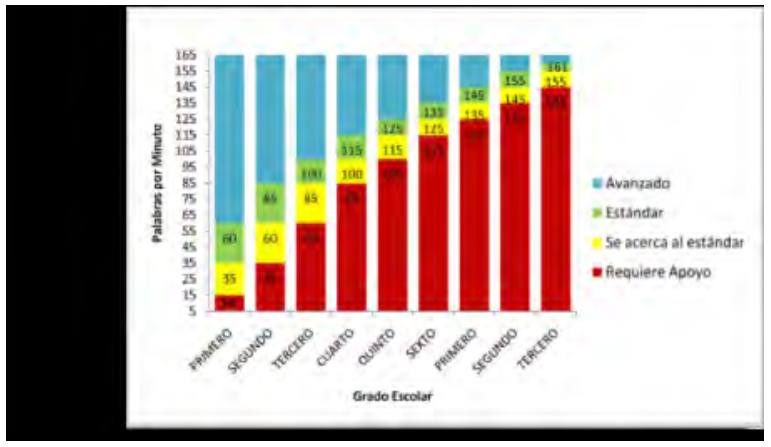

Fuente: http://www.sep.gob.mx/work/appsite/lectura.pdf

Estos estándares se tomaron en cuenta a pesar de que en los planes y programas de estudio de primero de primaria 2011 elaborados por la Secretaría de Educación Básica, establecen que no hay edad ideal para desarrollar la lectoescritura y que la edad ideal para empezar a medir la lectura de comprensión será hasta que al alumno haya terminado su segundo periodo de educación básica, que es al término del tercer año de primaria, entre los 8 y 9 años de edad (SEP, 2012).

Al trabajar de manera estadística el instrumento de investigación aplicado se puede observar que la fiabilidad del instrumento es superior a los .800 en la escala de Alfa de Cronbach por lo que sus resultados son confiables para su análisis.

\begin{tabular}{|c|c|}
\hline \multicolumn{2}{|c|}{ Estadísticos de fiabilidad } \\
\hline Alfa de Cronbach & N de elementos \\
\hline 0.948 & 7 \\
\hline
\end{tabular}

\begin{tabular}{|l|c|c|c|c|c|}
\hline \multicolumn{7}{|c|}{ Estadísticos descriptivos } \\
\hline & $\mathrm{N}$ & Mínimo & Máximo & Media & Desv. típ. \\
\hline VELOCIDAD LECTORA & 26 & 1 & 4 & 3.1923 & 1.16685 \\
\hline FLUIDEZ LECTORA & 26 & 1 & 4 & 2.9615 & 1.14824 \\
\hline PREGUNTAS GUIAS & 26 & 0.25 & 2 & 1.3846 & 0.58835 \\
\hline COMPRENSION LECTORA & 26 & 1 & 4 & 3.0769 & 1.09263 \\
\hline MEDIA GRAL. & 26 & 0.81 & 3.5 & 2.6538 & 0.96002 \\
\hline N válido (según lista) & 26 & & & & \\
\hline
\end{tabular}

En el análisis descriptivo de las dimensiones que integran el estudio y la media general se puede observar que en promedio el grupo de primero A de la primaria Real del Río vespertina obtuvo un 2.65, que redondeado se convierte en 3, basándose en la escala de la SEP es equivalente a que es un grupo que alcanza el Estándar en los niveles nacionales de lectura. Pero tratando de identificarse los puntos de mejora se detecta una dimensión en la cual se obtienen resultados bajos, tal es el caso de Las preguntas guías, en donde se alcanzó un 1.38, 
que redondeado baja a 1 y de acuerdo a la escala de la SEP este grupo requiere apoyo en ese rubro. Por lo anterior se va a profundizar en esta dimensión que arroja resultados que podrían tener puntos de mejora.

\begin{tabular}{|l|c|c|c|c|c|}
\hline \multicolumn{7}{|c|}{ Estadísticos descriptivos } \\
\hline p4 Qué le pidió Toño a su mamá & $\mathrm{N}$ & Mínimo & Máximo & Media & Desv. típ. \\
\hline $\begin{array}{l}\text { p5 Toño realmente es generoso como pensaba } \\
\text { su mamá }\end{array}$ & 26 & 0 & 2 & 1.58 & 0.578 \\
\hline $\begin{array}{l}\text { p6 El señor que gritaba en la calle ¿estaba } \\
\text { desesperado, como le dijo Toño a su mamá? }\end{array}$ & 26 & 0 & 2 & 1.31 & 0.838 \\
\hline p7 Ahora, cuéntame la historia que leíste & 26 & 0 & 2 & 1.12 & 0.711 \\
\hline N válido (según lista) & 26 & & & & 0.582 \\
\hline
\end{tabular}

En el cuadro de las preguntas que conforman la dimensión de Preguntas Guías se identifica que en la número 6 y 7 la media obtenida es de 1.31 y 1.12 respectivamente, que redondeadas a 1 y basándonos en la escala de la SEP, equivaldría a que los alumnos de primero A de la Escuela Primaria Real del Río vespertina de Mexicali no alcanzan a obtener dominio suficiente de las preguntas en donde más necesitan de profundidad para su explicación. Lo que podría indicar que los alumnos se les dificultan el estructurar la explicación de toda una historia. Tomándose las afirmaciones más bajas se hizo un análisis de Frecuencias

p6 EI señor que gritaba en la calle ¿estaba desesperado, como le dijo Toño a su mamá?

\begin{tabular}{|l|l|l|l|l|l|}
\hline \multicolumn{2}{|c|}{} & Frecuencia & Porcentaje & $\begin{array}{l}\text { Porcentaje } \\
\text { válido }\end{array}$ & $\begin{array}{l}\text { Porcentaje } \\
\text { acumulado }\end{array}$ \\
\hline \multirow{4}{*}{ Válidos } & 0 & 6 & 23.1 & 23.1 & 23.1 \\
\cline { 2 - 7 } & 0 puntos de crédito & 6 & 23.1 & 23.1 & 46.2 \\
\cline { 2 - 7 } & 1 punto de crédito & 14 & 53.8 & 53.8 & 100 \\
\cline { 2 - 7 } & Total & 26 & 100 & 100 & \\
\hline
\end{tabular}

\begin{tabular}{|l|l|l|l|l|l|}
\hline \multicolumn{2}{|c|}{ p7 Ahora, cuéntame la historia que leíste } \\
\hline \multirow{3}{*}{} & Frecuencia & Porcentaje & $\begin{array}{l}\text { Porcentaje } \\
\text { válido }\end{array}$ & $\begin{array}{l}\text { Porcentaje } \\
\text { acumulado }\end{array}$ \\
\hline \multirow{3}{*}{ Válidos } & 0 & 5 & 19.2 & 19.2 & 19.2 \\
\cline { 2 - 7 } & 0 puntos de crédito & 13 & 50.0 & 50.0 & 69.2 \\
\cline { 2 - 7 } & 1 punto de crédito & 8 & 30.8 & 30.8 & 100.0 \\
\cline { 2 - 7 } & Total & 26 & 100.0 & 100.0 & \\
\hline
\end{tabular}


Lo que se observa es que la mayor parte del grupo está en el campo de 1 crédito, es decir, que la mayoría pudo dar su respuesta cercana pero no lo suficientemente amplia como para asignarle los 2 créditos lo que significa que aún falta comprensión lectora para poder manejar de mejor manera la información. Finalmente en la pregunta en donde los alumnos debía de construir la explicación de todo el cuento por ellos mismos se observa es que la mayor parte del grupo está en el campo de 0 y 1 punto de créditos es decir que aún falta el manejo de la información de manera eficiente, los alumnos pudieron ir respondiendo a preguntas parciales, pero al pedirles que contaran la historia completa omitían algunas situaciones que en los Estándares Nacionales de Lectura se consideran necesarias para asignarle 2 créditos. Al analizar las dimensiones de velocidad lectora y fluidez lectora son las que más correlación tienen con la Media General es decir, que los alumnos que salieron con mejor velocidad y fluidez fueron los que al final arrojaron mejores resultados en el cumplimiento de los Estándares Nacionales de Lectura dentro del primer año A de la Escuela Primaria Real del Río vespertina, perteneciente a la zona II de primarias estatales en Mexicali.

\section{Conclusiones}

- Ante la variedad de escenarios internacionales presentados e investigaciones analizadas se puede afirmar que en México la Reforma Integral de Educación Básica 2009 cuenta con principios pedagógicos actualizados, pero no logró complementarlos con la realidad que se viven en muchas zonas de bajos recursos en el país.

- La educación debe descentralizarse en México, que las entidades federativas puedan rediseñar o adaptar los planes y programas a sus características geográficas y socioeconómicas traería beneficios tales como los obtenidos por programas de intervención desarrollados en países del extranjero.

- En el caso de la lectura de comprensión, es claro que los docentes están trabajando con vacío de conocimientos en la metodología que aplican para desarrollarla. Las escuelas formadoras de docentes deberán de reformarse, para que a los futuros docentes les presenten las orientaciones pedagógicas que respondan a la formación de los alumnos en las nuevas generaciones, pero que a la vez, cuenten con las herramientas metodológicas necesarias para trabajar con esas poblaciones en riesgo social, donde la alfabetización emergente y los ambientes motivadores no se dieron.

- La alfabetización temprana representa un factor determinante en la mayoría de las investigaciones citadas, los docentes deben tener en cuenta que hay que equilibrar el principio establecido en el plan y programas de estudio 2011 de la Secretaría de Educación Pública, en donde los alumnos son los constructores de su proceso de comprensión lectora, pues al dejar de lado el trabajo con el alfabeto y la relación grafía fonema, se ignora la base de conocimientos previos a la que recurrirá el alumno para seguir a lo más complejo.

- Es importante tener en cuenta que no es lo mismo trabajar con población promedio y población en situación social de riesgo. Los docentes que laboran en esos centros deberán de ser actualizados o agregar a su formación una metodología que pueda suplir a esa alfabetización emergente que no se dio en el contexto de sus alumnos. Para este hecho el método Global de Análisis Estructural responde a las necesidades antes mencionadas, pues es un proceso en el cual los alumnos analizan textos, se da la lectura en voz alta, el trabajo con oraciones, palabras y finalmente sílabas con sonidos específicos de la grafía. Es decir, con esto se le brindara al alumno la oportunidad de conocer lo elemental pero 
dentro de una pedagogía actualizada, acorde con las necesidades de las sociedades del conocimiento.

- Se pudo observar que dentro del grupo evaluado los alumnos con mayor velocidad y fluidez lectora fueron los que obtuvieron mejores resultados en su logro de los Estándares Nacionales de Lectura, es decir, que va implícito el número de palabras leídas por minuto, pues los alumnos que leyeron con más velocidad y fluidez obviamente fueron los que mayor número de palabras alcanzaron, lo que significa que el alumno que puede ir hilando la mayor cantidad de palabras dentro de un texto es el que mayor comprensión y significado obtendrá del mismo.

- En el apartado de preguntas guías fue curioso como en este grupo evaluado la complejidad de tratar de explicar de manera global el cuento leído fue mucha, pues la mayoría omitía algunos aspectos en diferentes etapas del texto, es decir, algunos dejaban de lado partes del desarrollo o personajes que no fueron significativos para ellos, y que dentro de los Estándares Nacionales de Lectura si se debían de contemplar. Pero sienta las bases necesarias para esperanzarse, con argumentos, que en el segundo grado de primaria los alumnos lograra una comprensión lectora completa.

- Que el grupo de primero A de la Escuela Primaria Real del Río vespertina de la zona II escolar de primarias en el municipio de Mexicali haya obtenido un promedio general de 3 (Estándar) en la escala de los Estándares Nacionales de Lectura, es de gran importancia, pues significa que es lo necesario que necesita el alumno para cumplir con los demás propósitos y objetivos que se establecen en el plan y programas de estudio de primer grado, y que servirá para los alumnos tengan bases sólidas para los siguientes grados escolares dentro de su educación básica.

- El que los alumnos de primer grado tengan una buena lectura de comprensión repercutirá en todas las asignaturas que complementan el currículo del primer ciclo, pues incluso para las matemáticas se necesita comprensión lectora, lo que puede ser una de las causas que también se tengan resultados negativos en el área de matemáticas en las evaluaciones nacionales.

- El trabajo con el método global de análisis estructural en ningún momento contravino con la metodología de la Reforma Integral de Educación Básica, al contrario, fue un complemento que sentó bases sólidas en los alumnos del grupo de primer año A de la Escuela Primaria Real del Río vespertina. 


\section{Referencias}

Abadzi, H. (2007). Aprendizaje eficaz y pobreza: Ideas desde las fronteras de la neurociencia. Santiago de Chile: Ediciones UCSH.

Acosta Luévano, R.M. (2007). La enseñanza de la lectura y escritura en el primer grado de primaria. Recuperado en http://www.upn011.edu.mx/publicaciones/revistas/UPNenlinea/0007.html, el 25 de abril de2012.

Araya Ramírez, J. (2009). La valoración del componente fonológico en niños que inician el proceso de lecto-escritura. (Spanish). Káñina,33(2), 33-41.

Araya, V., Alfaro, M. y Andonegui, M. (2007). Constructivismo: orígenes y perspectivas. Venezuela. Laurus, vol. 13, núm. 24, mayo-agosto, pp. 76-92, Universidad Pedagógica Experimental Libertador.

Arroyo Sarabia, M., de los Santos, L., Gasca García, G., \& Blanca Orozco Carro, R. (2010). Mejoramiento de la comprensión lectora basada en el aprendizaje colaborativo en la enseñanza media básica. (Spanish). Apertura: Revista De Innovación Educativa, 2(2), 36-47.

Ausubel, D. P. (1976). Psicología educativa. Un punto de vista cognoscitivo. Ed. Trillas. México.

Backhoff, E., Sánchez, A., Peón, M. y Andrade, E. (2010). Comprensión lectora y habilidades matemáticas de estudiantes de educación básica en México: 2000-2005. Revista Electrónica de Investigación Educativa, 12 (1). Consultado el día de mes de año en: $\mathrm{http}: / /$ redie.uabc.mx/vol12nol/contenido-backhoffsanchez.html

Bravo, L., Villalón, M. y Orellana, E. (2006). Diferencias en la predictividad de la lectura entre primer año y cuarto año básicos. Psykhe, 15(1), 3-11.

Carrasco, A. (2003). La escuela puede enseñar estrategias de lectura y promover su regular empleo. Revista Mexicana de Investigación Educativa, Vol. 8, N 17, enero-abril, pp.129-142.

Connor, C. M., Morrison, F. J. y Slominski, L. (2006). Preschool instruction and children's literacy skill growth. Journal of Educational Psychology, 98(4), 665-689.

De La Mata, M. L. (2003) Text remembering as a social process: the role of teacher-student interaction in the acquisition of structure strategy. Learning and instruction, 13, pp. 93-115.

Decroly, O. y Orellana Garrido J. (1934). Psicología aplicada a la educación. Madrid.

Díaz Barriga, Ángel. (2001). El debate por la lectura. Entre lo simple y la planeación. Perfiles Educativos, Vol. 23, No 92, pp.2-5, México.

Eyzaguirre, B. y Fontaine, L. (2008). Las Escuelas que tenemos. Santiago, Chile: Centro de Estudios Públicos.

Ferreiro, E. (1988). Nuevas perspectivas sobre los procesos de lectura y escritura. México. Ed. Siglo XXI.

Flavell, J. (1996). El desarrollo cognitivo. España, Prentice-Hall.

Flint, T. (2010). Making Meaning Together: Buddy Reading in a First Grade Classroom. Early Childhood Education Journal, 38(4), 289-297. doi:10.1007/s10643-010-0418-9

Foulin, J. N. (2005). Why is letter-name knowledge such a good predictor of learning to read?. Reading and Writing: An Interdisciplinary Journal, 18(2), 129-155.

García-Huidobro, J. E. (2006). Formación inicial de educadoras(es) de párvulos en Chile. Expansiva, Serie en Foco, 80, 1-26.

Hipwell, P., \& Klenowski, V. (2011). A case for addressing the literacy demands of student assessment. Australian Journal Of Language \& Literacy, 34(2), 127-146.

Justice, L. M., Mashburn, A., Hamre, B. y Pianta, R. (2008). Quality of Language and literacy instruction in preschool classrooms serving at-risk pupils. Early Childhood Research Quarterly, 23, 51-68.

López Oliveros, J. y Álvares Valles, J. (1991). Enseñanza de la lectura y psicología: análisis de los métodos sintéticos. Psicothema, vol. 3, no. 1, pp. 121-136.

López, M. (2009) Dificultades del profesorado en el proceso de identificación del alumnado con TDAH. Revista española de pedagogía, 67: 244, septiembre-diciembre, pp. 545-566. 
Madariaga, J., Chireac, S., \& Goñi, E. (2009). Entrenamiento al profesorado para la enseñanza de estrategias de comprensión lectora. (Spanish).Revista Española De Pedagogía, (243), 301318.

Martinez, E. Y Madariaga, J. M. (2006). Experiencia en torno a la enseñanza de estrategias de comprensión y metacomprensión lectora. Revista de Psicología General y Aplicada, 59, pp. 149164.

McKeown, M. G., y Beck, I. L. (2006). Encouraging language interactions with stories. En D. K. Dickinson y S. B. Neuman (Eds.), Handbook of early literacy research (pp. 281-294). New York: The Guilford Press.

National Early Literacy Panel (2008). Developing early literacy: Report of the National New York: The Guilford Press.

OCDE. (2009). Resultados de PISA en México. Recuperado el 25 de mayo de 2012 en: http://www.radioformula.com.mx/notas.asp? Idn $=144701$

OCDE/ Instituto Nacional de Calidad y Evaluación (INCE). (2000). Proyecto PISA. La medida de los conocimientos y destrezas de los alumnos: Un nuevo marco para la evaluación. Madrid.

Organización para la Cooperación y el Desarrollo Económico (2010). Education at a Glance: OCDE Indicators. París.

Piaget, J. (1974). A dónde va la educación. Barcelona: Ariel.

Poder Ejecutivo (2002). Decreto de creación del Instituto Nacional para la Evaluación de la Educación, publicado en el Diario Oficial de la Federación el día 8 de agosto de 2002.

Powell, D. R., Diamood, K. E., Bojczyk, K. E. y Gerde, H. K. (2008). Head Start teachers' perspectives on early literacy. Journal of Literacy Research, 40, 422-460.

Programa Estado de la Nación. (2007). CONARE San José, Costa Rica.

Programas de Estudios de Educación Básica. (2009). México, SEP

Ramos, J. (2004). Prueba fonológica PECO. Revista de los psicólogos de la educación, vol 11 Colegio Oficial de Psicólogos de Madrid, España.

Scarborough, H. S. (2001). Connecting early language and literacy to later reading (dis)abilities: Evidence, theory, and practice. En S. Neuman y D. Dickinson (Eds.) Handbook for research in early literacy (pp. 97-110). New York: Guilford Press.

SEP. (1993). Plan y programas de estudio. México. Conaliteg.

SEP. (2009). Reforma Integral de Educación Básica. Planes y programas. México. Conaliteg.

Shaw, P., \& McMillion, A. (2011). Components of success in academic reading tasks for Swedish students. Iberica, (22), 141-162.

Smith, P. H., Jiménez, R. T., \& Ballesteros Pinto, R. (2005). ¿Hay una pedagogía nacional de la lectoescritura? (Spanish). Lectura Y Vida, 26(1), 14-24.

Subsecretaría de Educación Básica. (2012). Finalidad de ENLACE. Recuperado el 15 de mayo de 2012 en: http://www.enlace.sep.gob.mx/que_es_enlacel

Taylor, J., \& Schatschneider, C. (2010). Genetic Influence on Literacy Constructs in Kindergarten and First Grade: Evidence from a Diverse Twin Sample. Behavior Genetics. 40(5), 591602. doi:10.1007/s10519-010-9368-7.

Tolchinsky, L. (2006). The emergence of writing. En C. A. MacArthur, S. Graham y J. Fitzgerald (Eds.), Handbook of Writing Research (pp. 83-95). New York: The Guilford Press.

Vellutino, F. R. y Scanlon, D. M. (2002). Emergent Literacy Skills, Early Instruction and Individual Differences as Determinants of Difficulties in Learning to Read: The Case for Early Intervention. En S. B. Neuman y D. K. Dickinson (Eds.), Handbook of Early Literacy Research (pp. 295-321). New York: The Guilford Press.

Villalón, M., Förster, C. E., Cox, P., Rojas-Barahona, C. A., Valencia, E., \& Volante, P. (2011). Resultados de la enseñanza de estrategias de lectura y escritura en la alfabetización temprana de niños con riesgo social. (Spanish). Estudios Sobre Educacion, (21), 159-179.

Vygotsky, L. S. (1978). Mind in society: Development of higher psychological processes. Cambridge, MA: Harvard University Press. 
Zill, N. y Resnick, G. (2006). Emergent literacy of low-income children in Head Start: Relationships with child and family characteristics, program factors and classroom quality. En D. K. Dickinson y S. B. Neuman (Eds.), Handbook of early literacy research (pp. 41-51). New York: The Guilford Press.

\section{Sobre el Autor}

Maestro Leonardo Cíntora: Soy el Mtro. Leonardo Abdul Cíntora Castañeda, Licenciado en Educación Primaria por la Benemérita Escuela Normal Urbana Federal Fronteriza, Maestro en Pedagogía por la Universidad Estatal de Estudios Pedagógicos y actualmente soy doctorando en Educación. Docente en la II Zona Escolar de Primarias. Mi interés es encontrar formas de trabajo que respondan a las necesidades de la población que estoy atendiendo, para brindarles a los alumnos herramientas sólidas para su ingreso y desempeño en las sociedades del conocimiento. 